\title{
Performance of Antenna Selection in MIMO System Using Channel Reciprocity with Measured Data
}

\author{
Peerapong Uthansakul, Nattaphat Promsuwanna, and Monthippa Uthansakul \\ School of Telecommunication Engineering, Suranaree University of Technology, Muang, Nakhon Ratchasima 30000, Thailand \\ Correspondence should be addressed to Nattaphat Promsuwanna, jame_maxwell@hotmail.com
}

Received 15 February 2011; Revised 1 April 2011; Accepted 5 April 2011

Academic Editor: Byungje Lee

Copyright () 2011 Peerapong Uthansakul et al. This is an open access article distributed under the Creative Commons Attribution License, which permits unrestricted use, distribution, and reproduction in any medium, provided the original work is properly cited.

\begin{abstract}
The channel capacity of MIMO system increases as a function of antenna pairs between transmitter and receiver but it suffers from multiple expensive RF chains. To reduce cost of RF chains, antenna selection (AS) method can offer a good tradeoff between expense and performance. For a transmitting AS system, channel state information (CSI) feedback is required to choose the best subset of available antennas. However, the delay and error in feedback channel are the most dominant factors to degrade performances. In this paper, the concept of AS method using reciprocal CSI instead of feedback channel is proposed. The capacity performance of proposed system is investigated by own developing Testbed. The obtained results indicate that the reciprocity technique offers a capacity close to a system with perfect CSI and gains a higher capacity than a system without AS method. This benefit is from 0.9 to $2.2 \mathrm{bps} / \mathrm{Hz}$ at SNR $10 \mathrm{~dB}$.
\end{abstract}

\section{Introduction}

A multiple-input-multiple-output (MIMO) system recently becomes one of the most attractive techniques for the future use because it proposes an extensive improvement over conventional smart antenna systems in both quality of service (QoS) and the transfer rate [1-4]. However, using multiple antennas requires multiple radio frequency (RF) chains which consist of amplifiers, up and down converters, digital to analog converters, and so forth. Those are typically very expensive. A promising approach for reducing cost while retaining a reasonably large fraction of the high potential data rate of an MIMO approach is to employ some form of antenna selection (AS) [5-8]. The AS method employs a reduced number of RF chains at the receiver (or transmitter) and attempts to optimally allocate each chain to one of a large number of receiving (transmitting) antennas which are usually cheap elements. In this way, only the best set of antennas is used while the remaining antennas are not employed, thus reducing the number of required RF chains. It can be noted that water-filling technique always provides the optimal performance which is better than antenna selection only if the numbers of RF chain of both systems are the same. However, water-filling technique requires the full equipped RF modules for each branch of MIMO antennas which affect the price of manufacturing considerably. To reduce RF hardware by using antenna selection technique is more economic. Thus, somewhere in the middle, an effective MIMO antenna selection can achieve a good tradeoff between performance and cost.

In literatures [9-17], the developments of AS method are classified into two main topics. At first, the algorithms to select the best subset of antennas are on focus. These algorithms can be applied to either transmitter [9] or receiver [10]. The fast and precise selections are the required demand in practice. However, the success of these algorithms depends on the knowledge of channel state information (CSI) especially for the transmitting AS system that CSI feedback is necessarily required to choose the best subset of antennas [11-14]. Although the work presented in [15] tries to perform AS method without knowing CSI at transmitter for transmitting AS system, the expense of many iterations degrades its attraction.

For second topic, researchers pay attention to the methods of CSI acquisitions. This is because the more exact CSI is realized, the more enhanced performance of AS method 
is obtained. Unfortunately, the CSI is usually not available at the transmitter. So the method to realize CSI is still important. In literatures, there are two approaches in order for the transmitter to obtain the CSI. The first approach utilizes CSI from feedback channel and the second approach is based on the reciprocity principle. In the first method, the forward channel is estimated at receiver and then it is sent back to the transmitter through the reverse channel. This method does not function properly if the channel is rapidly changed. In order to realize the correct CSI at transmitter, more frequent estimations and feedbacks are required. As a result, the overheads for the reverse channel become prohibitive. In turn, the second approach based on reciprocity does not have such a problem. Due to the reciprocity principle, it is well known that the radio propagation channel is reciprocal between two antennas. Ideally, the forward and reverse channels are assumed to be the same. Therefore, the transmitter can realize the forward CSI by estimating the reverse CSI instead. In timedivision-duplex (TDD) mode, the same carrier frequency is alternately used in forward and reverse channels. The propagation surrounding is not rapidly changed by time. So the channel coefficients are able to be considered as similar for both directions. Based on TDD mode, the reciprocity approach is superior to any explicit feedbacks.

Recently, there have been many researches concerning channel reciprocity of an MIMO system which is based on the nonreciprocal effects between forward and reverse channels caused by any mismatches among RF components and interferences between transmitter and receiver $[16,17]$. However, from all works described in literatures $[16,17]$, the system model is based on the assumption that the forward and reverse channels are exactly identical. This assumption is not valid in practice because the transmitter realizes the reciprocal channel after the time that signals used for estimating channel completely passes through it. Also the time-varying noises on forward and reverse channels are not identical. Therefore, apart from component mismatches and interferences, the time delays and noises between expected and actual channels cause the nonreciprocal effect in practice. As a result, it is interesting to examine whether AS method still works under such a deviation of nonreciprocal CSI.

In this paper, the performances of adaptive MIMO system with AS method at transmitter based on channel reciprocity are investigated. The data are measured and tested by our developing Testbed based on FPGA board. In recent times, most researchers move their experiments from simulations into real measurements. MIMO Testbed [18-23] is one the most comfortable platforms to realize the true performance of a proposed system under a real circumstance. For the work presented in [18-20], the performance investigations of MIMO system under indoor and outdoor have been reported through the Testbed. In [23], a transmitting AS system with an eigenbeam for MIMOOFDM system is employed. This work achieves CSI via feedback technique and uses it to compute eigenvectors for selecting the best subset of transmitting antennas. In summary, all MIMO Testbeds presented in literatures utilize
CSI from feedback channels. Moreover, some works [21, 22] use a direct link to perform feedback channels which exclude any errors due to wireless operations. As far as the survey of the authors is concerned, the issue of channel reciprocity for MIMO Testbed has never been reported in any publications. Hence, the contributions of this paper mainly fall into two issues. Firstly, the use of channel reciprocity for AS method in a MIMO system is originally demonstrated. The second contribution is on a proposed MIMO Testbed working by FPGA processors which is ready to be launched as commercial products. More importantly, it is interesting to delete the need of feedback channels by replacing reciprocity technique because this can save costs of system complexity and make the system more reliable.

In this work, the effect of the mismatches of RF components can be assumed to be neglected by using the same components at both transmitter and receiver. The CSI information between transmitter and receiver used in AS method is acquired by channel emulator in which forward and reverse CSIs are measured from real propagation environments. The $2 \times 4$ MIMO channels are considered as $2 \times 2$ by AS method and then the channel capacity is calculated by computer to find the optimal subset of antennas. In addition, the comparison between feedback approach and reciprocity approach are also undertaken to provide the fair judgment with measured data. In [24], the authors performed the initial study to confirm the reciprocity approach. However, many data details are missing and have been fulfilled in this paper. More data measurements have been undertaken. In particular, the new discussion on measured coherent time is added to practically prove the use of reciprocity. The results in this paper are helpful to realize the use of channel reciprocity in practice and its impact on channel capacity due to nonidentical CSI between forward and reverse channels. The remainder of this paper is organized as follows. In Section 2, the MIMO system model and two approaches estimating CSI are presented. Section 3 describes the channel measurement and then the testing implementation is detailed in Section 4. The MIMO channel capacities are discussed in Section 5. Finally, the paper conclusion is given in Section 6.

\section{MIMO System Model}

2.1. MIMO Channel Capacity. Consider the MIMO system which has $N_{T}$ transmitting antennas and $N_{R}$ receiving antennas. The formula of MIMO channel capacity is given in (1) [4]. This expression presents the averaging capacity in $\mathrm{bps} / \mathrm{Hz}$ by assuming the ergodic process for channel matrix $\mathrm{H}$;

$$
C=E_{H}\left\{\log _{2} \operatorname{det}\left(\mathbf{I}_{N_{R}}+\frac{P_{T}}{P_{N} N_{T}} \mathbf{H H}^{*}\right)\right\},
$$

where $\mathbf{I}_{N_{R}}$ is the $N_{R} \times N_{R}$ identity matrix, $P_{T}$ is the total transmit power, $P_{N}$ is the noise power, $N_{T}$ is the number of transmitting antennas, $N_{R}$ is the number of receiving antennas, $E_{H}\{\}$ is the expectation over $\mathbf{H}$, and $*$ denotes the conjugate and transpose operation. 
When AS method is employed at the transmitter or the receiver, a subset of transmitting or receiving antenna elements is chosen. The channel seen by the subset is the sub-matrix $\mathbf{H}_{\text {sub }}$ which is obtained by selecting only the rows and columns of $\mathbf{H}$ that correspond to the selected receiving and transmitting antenna elements. The optimal subset is the one that leads to the largest mutual information between the antenna elements. The capacity of MIMO system with AS is given by

$$
C_{\text {sel }}=\max _{S(\mathbf{H})} \log _{2} \operatorname{det}\left(\mathbf{I}_{L_{R}}+\frac{P_{T}}{P_{N} L_{T}} \mathbf{H}_{\text {sub }} \mathbf{H}_{\text {sub }}{ }^{H}\right),
$$

where $\mathbf{I}_{L_{R}}$ is the $L_{R} \times L_{R}$ identity matrix, $\mathbf{H}_{\text {sub }}$ is a $L_{R} \times L_{T}$ matrix obtained by removing $N_{R}-L_{R}$ rows and $N_{T}-L_{T}$ columns from $\mathbf{H}$, and $S(\mathbf{H})$ denotes the set of all possible $L_{R} \times L_{T}$ sub-matrices of $\mathbf{H}$ that can be chosen. $L_{R}$ and $L_{T}$ represent the number of receiver RF chains and transmitter $\mathrm{RF}$ chains, respectively.

2.2. CSI Realization at Transmitter. As seen in Section 2.1, the system can achieve the optimal selection by employing the knowledge of the forward channel if there is no error of knowledged channel (perfect CSI). To obtain the CSI at transmitter, there are two approaches explained as follows.

2.2.1. Feedback Approach. For this approach, the receiver realizes a current CSI by channel estimation and uses the estimated channel to extract the data and to generate the feedback CSI. The feedback CSI is sent back to the transmitter using the feedback control channel. The transmitter, in turn, uses this information to customize the transmitted signal for the channel.

In practice, errors from feedback link which influence channel knowledge can degrade the capacity performance which is more pronounced when feedback link contains errors excessively. Under this assumption, the available CSI at transmitter can be expressed as

$$
\mathbf{H}_{T}=\mathbf{H}_{F}+\varepsilon_{E}+\varepsilon_{F},
$$

or

$$
\mathbf{H}_{T}=\mathbf{H}_{\mathrm{ES}}+\varepsilon_{F},
$$

where $\mathbf{H}_{F}$ is the forward channel, $\mathbf{H}_{T}$ is the available CSI at transmitter, $\varepsilon_{E}$ and $\varepsilon_{F}$ are $N_{R} \times N_{T}$ error matrix from estimation and error matrix from feedback link, respectively, and $\mathbf{H}_{\mathrm{ES}}$ is the channel matrix which is achieved by channel estimation.

2.2.2. Reciprocity Approach. According to the principle of reciprocity, the forward and reverse channels are identical when the time, frequency, and antenna locations are the same. Based on this principle, the transmitter may use the CSI obtained by the reverse link for the forward link. In practice, the forward and reverse channels are not truly identical because of the effect of channel fading, noises, and environments. The CSI known at transmitter can be given by

$$
\mathbf{H}_{T}=\mathbf{H}_{R}^{T}+\varepsilon_{E}+\varepsilon_{R},
$$

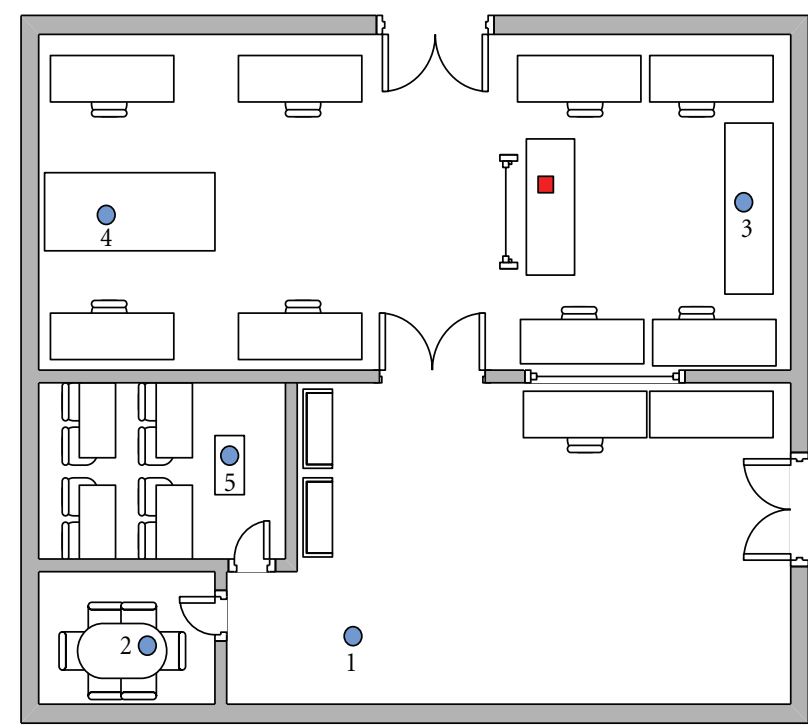

Network analyzer

Test point

Figure 1: Map of a measured area.

where $\mathbf{H}_{R}^{T}$ is the transpose of reverse channel matrix [25] and $\varepsilon_{R}$ is the $N_{R} \times N_{T}$ channel reciprocity error matrix realized by measurements.

\section{Channel Measurement}

The configuration of $2 \times 4$ MIMO system consists of network analyzer, power amplifier (PA) module, low-noise-amplifier module (LNA), and monopole antennas with $5 \mathrm{dBi}$ gain. The PA module is used at transmitter to increase transmitted power. The LNA module is used to increase received signal level. The channel coefficients in both magnitude and phase are measured by network analyzer. The center frequency is $2.45 \mathrm{GHz}$ and its bandwidth is $100 \mathrm{MHz}$. The data was measured by 5 times per location. In each time, 100 samples of channel data were recorded continuously within 1.5 seconds. However, we did not continue recording all 5 times in one round. The sequence of recording starts from Location 1 (100 samples) and then moves to Location 2 (100 samples) until Location 5 (100 samples). This sequence is called one set. In the first day, we performed two sets of measurements and the other three sets were done on the second day. In summary, 500 samples per location were collected over two days. In order to mitigate the effect of measurement noises, the function of network analyzer, called smoothing command, is used to average the measured data over specific time. In this work, the specific time is the default operation at $10 \mathrm{~ms}$. It means that each recorded sample is an averaging result over $10 \mathrm{~ms}$.

For the measured area, we choose large office room to provide many cases of study. Figure 1 shows the map of an office room. The circular markers refer to the locations where the measurement is undertaken. There are five measured 


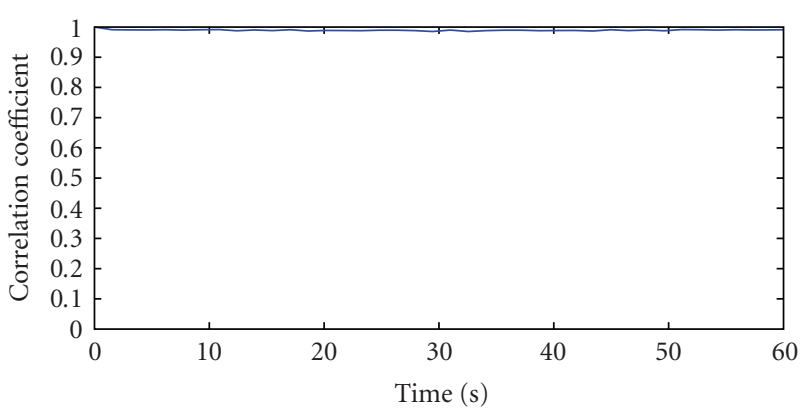

FIGURE 2: Correlation coefficients as a function of time in measured environment.

locations. For channel coefficient measurement, the forward channel and reverse channel are made by switching all modules (PA and LNA) but not including the cables. This is because all phase shifts and amplitude attenuations of cables are calibrated before measuring. While modules are being switched, all cables are placed at the same positions as they are started. Hence, by keeping the same configuration of cables for both forward and reverse channels and switching only PA and LNA modules, we believe that the measured results might reflect the true forward and reverse comparison. For PA and LNA modules, they are put in the same small boxes covered by absorbers and placed on fixed locations behind antennas array to reduce their fading effects.

The switching time between transmitter and receiver is at least 20-30 seconds. In order to confirm whether reciprocity channel is still valid for our measurements, the authors have calculated the correlation coefficient from the measured data as the same method as presented in [26]. The result of correlation coefficient as a function of time is shown in Figure 2. It is noticed that our result is similar to the result illustrated by static channel in [26]. In literatures, the coherent time achieved by experiments is defined as the duration from start to the time that correlation coefficient is lower than 0.5 . Therefore, it can be concluded that our measurements are performed under a static channel with a very large coherent time (over 20-30 s). Accordingly, the coherence time in our experiments is large enough to hold on the property of channel reciprocity.

Figure 3 shows an example of each element of $2 \times 4$ channel matrix at Location 4 , where $H_{i j}$ refers to the channel coefficient of $i$ th receiving antenna and $j$ th transmitting antenna. For the sake of illustration, it is noted that the symbol of $H_{i j}$ is referred as an entry element of only forward channel matrix while it is also referred as the transpose element of reverse channel matrix [25]. It can be observed that both forward and reverse channels are quite similar but not exactly the same. The amplitude deviation is about $\pm 2 \mathrm{~dB}$ and the phase deviation is about $\pm 15^{\circ}$. These deviations were ignored in all works presented in literatures $[16,17]$. The result is important to realize how these deviations influence the practical performance. As seen in Figure 3, the variation of measured data is very small because the environments seem to be a static channel. Hence, the results are grouped into two clusters, forward and reverse channels. However, the forward and reverse channels are not identical as expected because the measured data are collected in different time. For other locations, the deviations of amplitude and phase are similar to Location 4.

In addition, the correlation coefficients evaluated from measurements are $0.62,0.33,0.90,0.52$, and 0.73 for Locations 1, 2, 3, 4, and 5, respectively. Assuming all channel matrices of each location have the equally received SNR, thus it can be concluded that Location 2 is the most suitable area for MIMO operation because it yields the lowest correlation coefficient in comparison with other locations. Then, it is expected that Location 2 should offer more capacity than other locations as well. In turn, the capacity performance of Location 3 is expected to be poor due to its high correlation.

\section{Testbed Implementation}

4.1. Antenna Selection (AS) Technique. The capacity of MIMO system employing AS technique is described in (2); however this work concerns only the case of AS known at the transmitter. In order to find the optimal subset from knowing only CSI at transmitter modeled in (3) and (5) for feedback and reciprocity approaches, respectively, the conventional technique is applied by searching all possible subsets of antennas and then selecting the best subset providing the highest capacity. As a result, the formula of MIMO channel capacity with AS technique at transmitter can be given by

$$
C_{\text {sel }}=\max _{S\left(\mathbf{H}_{T}\right)} \log _{2}\left[\operatorname{det}\left(I_{L_{R}}+\frac{p}{L_{T}} \mathbf{H}_{T, \text { sub }} \mathbf{H}_{T, \text { sub }}{ }^{H}\right)\right] .
$$

where $\mathbf{H}_{T \text {, sub }}$ is the $N_{R} \times L_{T}$ sub-matrix (AS at transmitter) of CSI $\left(\mathbf{H}_{T}\right)$ obtained by feedback or reciprocity approaches.

It must be noticed that by using CSI in (6) to find the optimal subset of antenna, the differences between $\mathbf{H}_{T}$ and $\mathbf{H}\left(\mathbf{H}_{T} \neq \mathbf{H}\right)$ cause direct effect to the capacity performance in (2) due to implementing errors from either feedback or reciprocity channels. In this work, the exhaustive search is applied to find the best antenna subset from all possible cases. Although there are many algorithms proposed in the literature to select the antenna subset, the best solution is still the same as the one obtained from exhaustive search. The only benefits of other algorithms are fast processing and low complexity. The purpose of this work is to investigate the performance of reciprocity channels in comparison with feedback channels. So the same conclusion should be found for any AS methods.

4.2. Design of Developing Testbed. This work chooses field programmable gate array (FPGA) technology to implement $2 \times 4$ MIMO Testbed because FPGAs processing is introduced as promising alternative to custom ICs for implementing entire system on one chip and to provide flexibility of reprogram ability to the user. All functions are constructed inside FPGA boards including the AS method at transmitter where 2 transmitting antennas are selected. Hence, the system requires only 2 transmitting and 2 receiving components such as analog-to-digital converter 


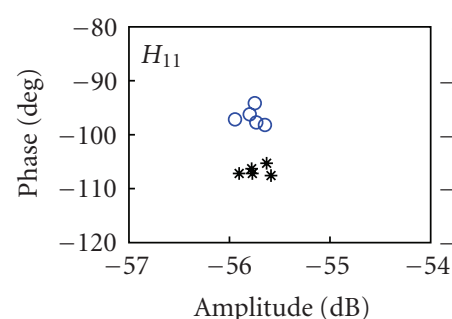

- Forward

* Reverse

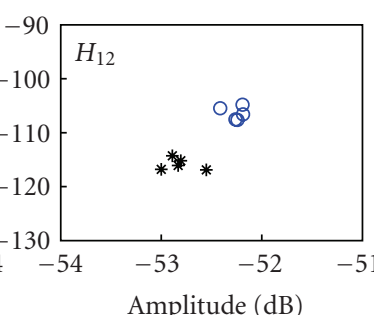

Forward

* Reverse

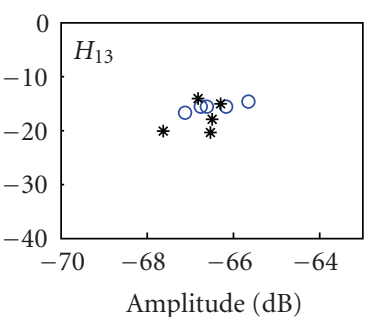

O Forward

* Reverse

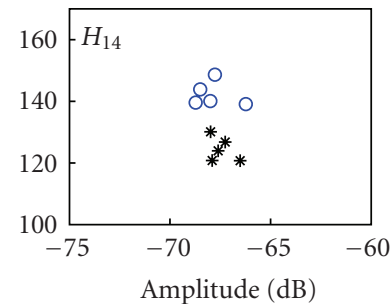

O Forward

* Reverse

(a)
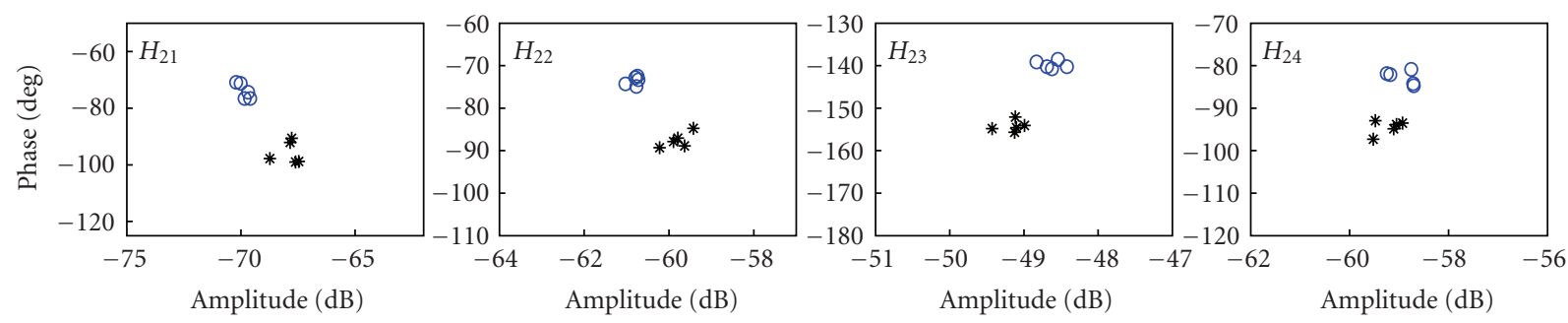

Forward

Forward

O Forward

- Forward

* Reverse

* Reverse

* Reverse

* Reverse

(b)

Figure 3: Example of forward and reverse channels measured at Location 4.

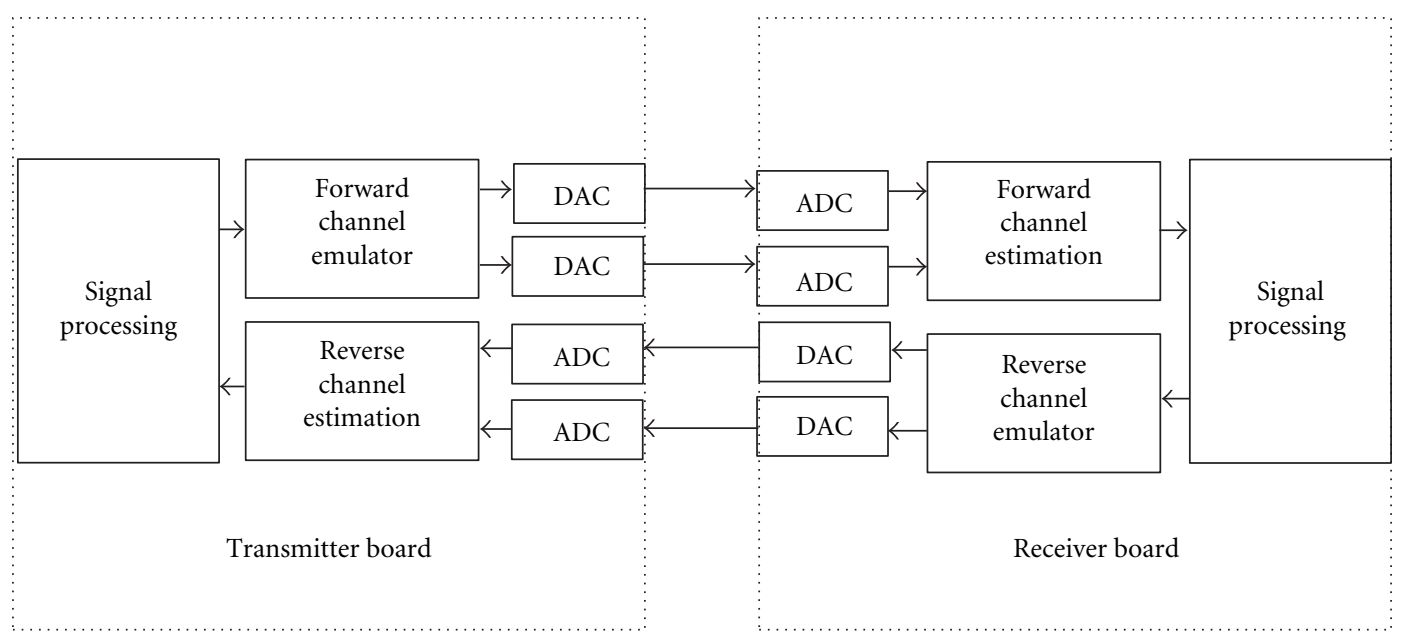

FIGURE 4: System diagrams of transmitter and receiver.

(ADC) and digital to analog converter (DAC). The block diagram of FPGA boards can be shown in Figure 4. As seen in Figure 4, RF components of transmitter and receiver have been replaced by using channel emulator. The concept of channel emulator has been adopted in many publications $[21,22]$ in order to simulate the various conditions of channel collected by real measurements. Another point of using channel emulator is to save cost of RF components because the boards can be functionally tested before passing through the production line.

In this work, we use Spartan 3An starter kit boards from Xilinx Company to implement transmitter and receiver which is explained as follows. Note that both transmitter and receiver boards shown in Figure 4 have the same components as transmitter and receiver to perform full duplex communication. However, we name the direction 
from transmitter board to receiver board as forward channel and the other direction as reverse channel.

4.2.1. Transmitter. The series of bit information ( 4 bits) are generated and then it is converted from series to parallel bit information. After that, it is modulated by BPSK modulation with frequency $12.5 \mathrm{kHz}$ in which bit 1 and bit 0 are represented by phase $0^{\circ}$ and $180^{\circ}$, respectively. The BPSK signal is fed to the channel emulator which acts as wireless communication channel. Finally, the signals from channel emulator are transformed to analog signals which are sent to receiver board.

4.2.2. Receiver. The received analog signals from transmitter are fed to the channel estimation block to estimate the CSI which is used to select the optimum subset of antennas in AS method. The estimated CSI is shown via chip scope pro software on PC which is connected to the receiver board.

4.3. Channel Estimation Method. To obtain CSI at both transmitter and receiver, we develop a simple technique to estimate CSI and it can reduce the complexity of hardware implementation. Consider MIMO system which has 4 transmitting antennas and 2 receiving antennas. The set of training sequence is specified by 5 times of four bit transmissions. These are $0111_{t=5}, 1011_{t=4}, 1101_{t=3}, 1110_{t=2}$, and $0000_{t=1}$ where $t$ indicates the order sequence. To understand the principle of channel estimation, first we have to understand the layout of communication in $2 \times 4 \mathrm{MIMO}$ which can be written as

$$
\left[\begin{array}{l}
y_{1} \\
y_{2}
\end{array}\right]=\left[\begin{array}{llll}
h_{1,1} & h_{1,2} & h_{1,3} & h_{1,4} \\
h_{2,1} & h_{2,2} & h_{2,3} & h_{2,4}
\end{array}\right]\left[\begin{array}{l}
x_{1} \\
x_{2} \\
x_{3} \\
x_{4}
\end{array}\right] .
$$

Then the received signal at each receiving antenna at $t$ time duration can be shown as

$$
\begin{aligned}
& y_{1}(t)=h_{1,1} x_{1}(t)+h_{1,2} x_{2}(t)+h_{1,3} x_{3}(t)+h_{1,4} x_{4}(t), \\
& y_{2}(t)=h_{2,1} x_{1}(t)+h_{2,2} x_{2}(t)+h_{2,3} x_{3}(t)+h_{2,4} x_{4}(t) .
\end{aligned}
$$

Let $S_{0}$ and $S_{1}$ be defined as BPSK signals representing 0 and 1 bit modulation, respectively. Then, the channel coefficient can be calculated by

$$
h_{k, t-1}=\frac{y_{k}(t=1)+y_{k}(t)}{2\left|S_{0}\right|} .
$$

In this work, (9) is implemented on FPGA to estimate both forward and reverse CSI. Accordingly, these estimated CSI are used in AS algorithm mentioned earlier.

4.4. Test Results. To complete testing system, channel emulators are operated for both forward and reverse channels. The channel information is determined by real measured data and programmed on FPGA boards. The obtained results are collected for the case of AS method, which is employed at the transmitter only. Personal computers are connected to the transmitter and receiver to provide programmable interface and capture all concurrence data. Moreover, the oscilloscope is used to capture a real signal coming from any concerned ports in order to compare signals between forward and reverse channels.

To implement channel emulators for both forward and reverse channels, the measured data described in Section 3 are used. However, channel information matrices from measurements have to be multiplied and divided by a constant value in order to adjust a suitable level for ADC and DAC specifications. This is to avoid the unwanted effect due to low sensitivity of ADC or DAC, which causes a signal error at receiver. It is noted that multiplying or dividing channel information matrices with a constant value does not change the property of channel characteristics.

Figure 5 shows an example of comparison in complex form between forward and estimated forward channel matrix using one sample of channel data at Location 1. The estimated forward and forward channels are similar in terms of real and imaginary parts. Although the deviations are still valid, these errors are very small when compared with errors between forward and reverse channels depicted in Figure 3. Therefore, the channel estimation implemented in the Testbed works very well and ensures the correct achievement of CSI from available sources, either feedback or reciprocity approaches.

\section{Results and Discussions}

5.1. Simulation Results. All capacity results in this section are off-line produced on computer by using real AS outcomes from Testbed selections mentioned in the previous section. Therefore, these capacities can be compared with various system conditions including Rayleigh propagation channel and perfect CSI system which are hardly measured in real scenarios. The MIMO channel capacities are computed by MATLAB programming when AS methods are employed by using (2) and (6). Note that $\mathbf{H}_{T}$ of forward and reverse channels are obtained by Testbed system. For feedback approach, at first we assume that there is no feedback error in feedback channel and then at the end of this section, the effect of this error will be illustrated. The simulations disregard the mismatches of RF circuits in transmitting/receiving components as well as mutual coupling effects because they are included in one part of channel measurements.

The capacity performances are based on cumulative distribution function (CDF) at $\mathrm{SNR}=10 \mathrm{~dB}$ by using 500 samples of the collected channel data and they are illustrated into five cases, Rayleigh channel, AS with feedback channel, AS with perfect CSI, AS with reciprocity channel, and fixed antennas. In case of fixed antennas, the first and second transmitting antennas are used to transmit signals for any locations. For the sake of comparison, the channels are normalized to provide a comparable discussion by $\sum\left|H_{i j}\right|^{2}=N_{T} N_{R}$ which limits total channel energy to one constant value. This normalization is done in order to compare channel properties between various conditions by 


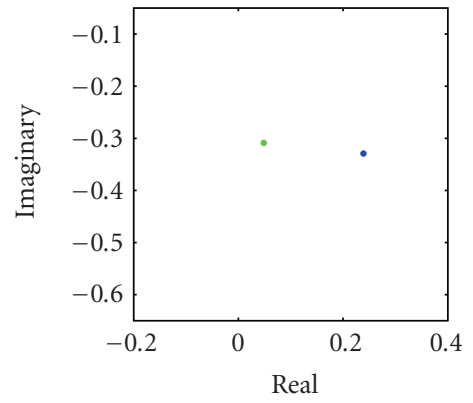

- Forward channel

- Estimated forward channel

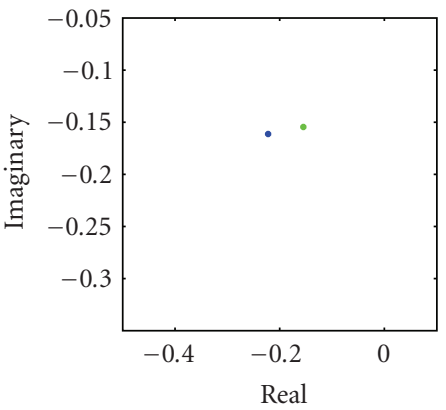

- Forward channel

- Estimated forward channel

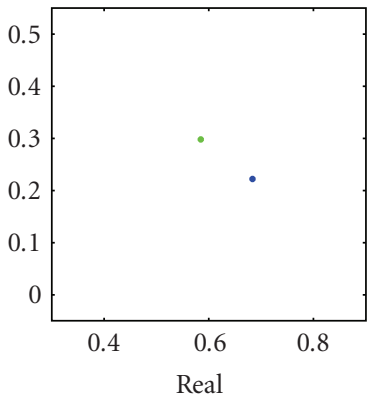

- Forward channel

- Estimated forward channel
- Forward channel

- Estimated forward channel

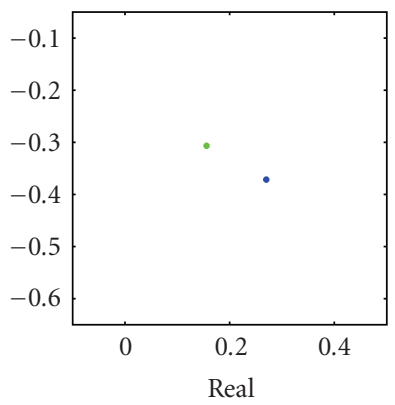

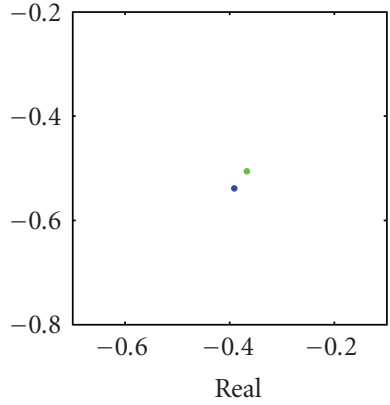

- Forward channel

- Estimated forward channel

(a)

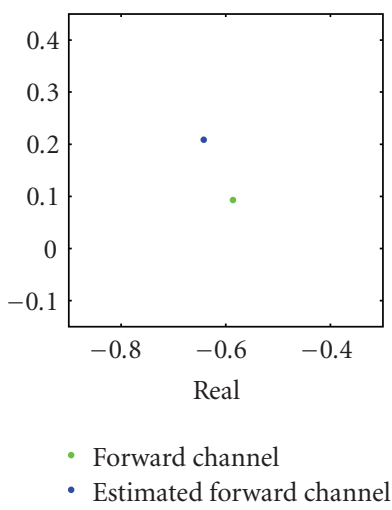

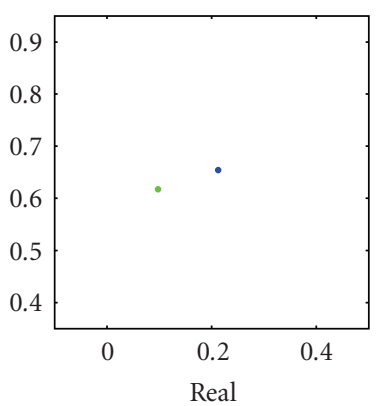

- Forward channel

- Estimated forward channel

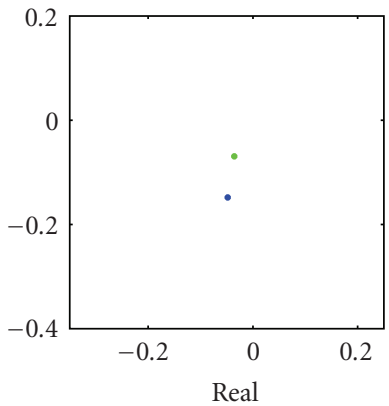

- Forward channel

- Estimated forward channel

(b)

FIGURE 5: Comparison example between forward and estimated forward channels at Location 1.

neglecting the effect of path loss. All details of five cases are explained as follows.

(i) Rayleigh Channel: this case represents the capacity of $2 \times 2$ MIMO system when wireless channel acts as Rayleigh fading channel which is random over 10,000 times.

(ii) Fixed Antennas: this case represents capacity of $2 \times 2$ MIMO system where the first and second antenna elements are used to transmit signals.

(iii) AS with feedback: this case represents the capacity when the CSI from feedback approach is used to select transmitting antennas in AS technique. Two of four transmitting antennas are optimally chosen to offer the best capacity.

(iv) AS with Perfect CSI: this case represents the capacity when the CSI is assumed to be perfectly known at transmitter. Hence, the selected transmitting antennas in AS technique are ideally optimal corresponding with the wireless channels. There is no error taken into account so that the capacity is computed by using (2).

(v) AS with reciprocity: this case represents the capacity when the CSI from reciprocity approach is used to select transmitting antennas in AS technique.
As mentioned in Section 3, there are five measured locations in which the channels are collected. The surrounding of each location is so different that the capacity results are considerably separated into each location to make a fair judgment on all approaches.

Location 1. As seen in Figure 6, the cumulative distribution function of capacity for AS methods with reciprocity and feedback approaches are close to a perfect CSI case where a feedback case is slightly better than a reciprocity case. In addition, AS method with reciprocity gives a performance gain $1.15 \mathrm{bps} / \mathrm{Hz}$ higher than a case of fixed antennas at $50 \%$ probability. However, AS method provides the capacity performance at $50 \%$ probability equal to a case of Rayleigh at $92 \%$ probability. This is because the channels at Location 1 offer AS method to select the antenna subset that causes a very low channel correlation.

Location 2. In Figure 7, the cumulative distribution function of capacity for fixed antennas, AS methods with reciprocity, and feedback are close to perfect CSI and they provide capacity at 50\% probability equal to a case of Rayleigh at $90 \%$ probability. It is interesting to observe that fixed antennas method provides a performance close to a perfect CSI case at this location. The reason is that fixed antennas method 


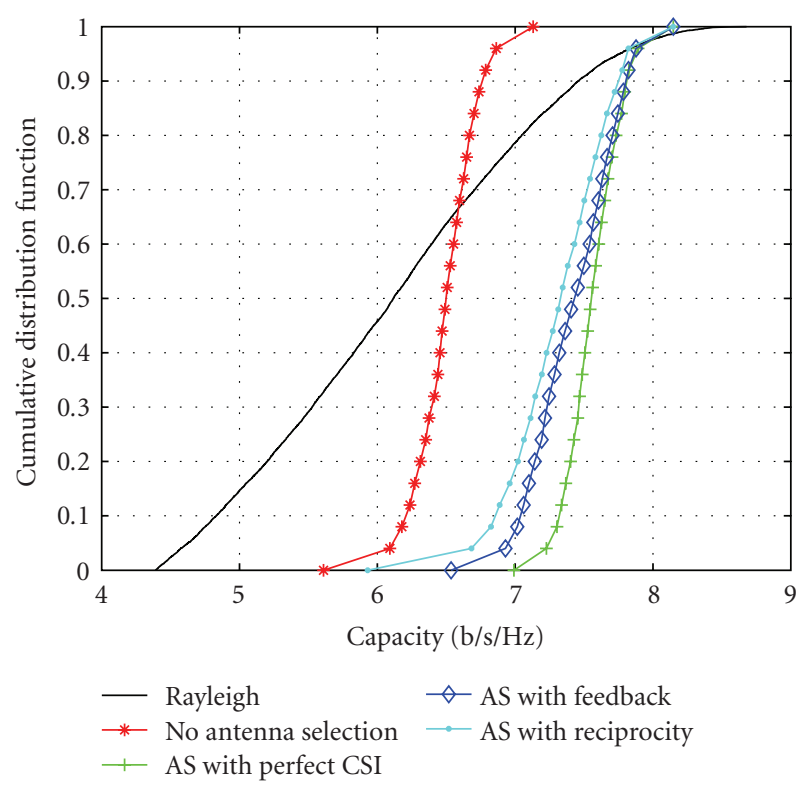

Figure 6: The cumulative distribution function of capacity at Location 1.

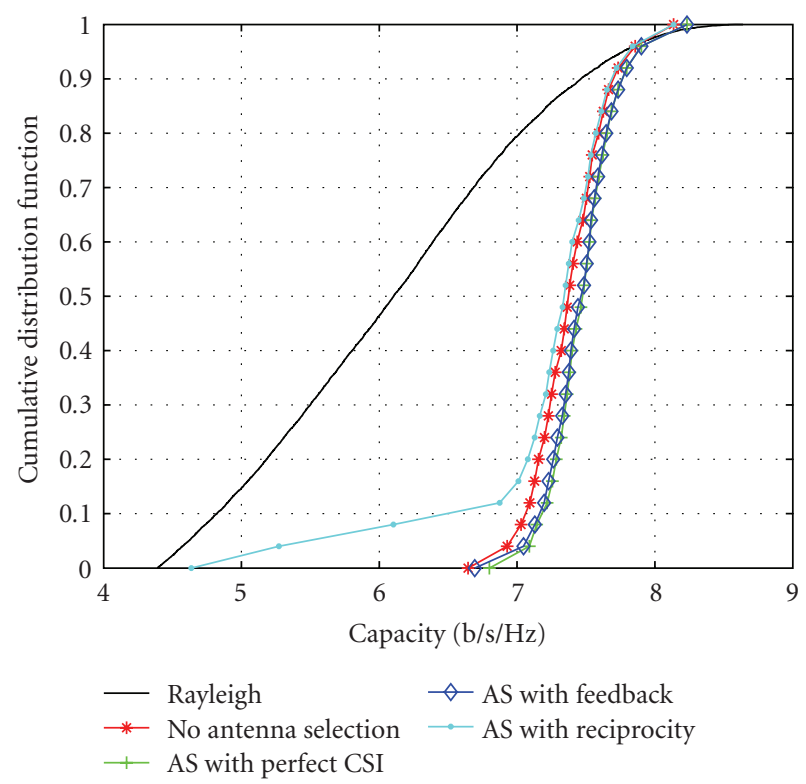

Figure 7: The cumulative distribution function of capacity at Location 2.

always fixes the first and second transmitting antennas to operate a $2 \times 2$ MIMO system which naturally becomes the best subset of antenna selections for this location.

Location 3. Figure 8 shows the cumulative distribution function of capacity at Location 3. In this location, the interesting point is that the average capacities of AS methods with any cases appear in the lower bound of Rayleigh while they exhibit in the upper bound for the other locations. It is also noticed that the performance of a reciprocity case seems to fail on selecting the best subset because the average deviation
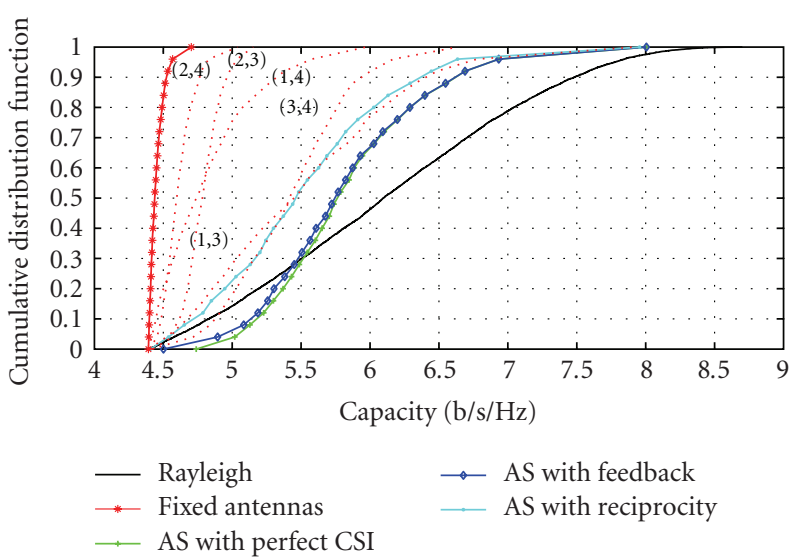

FIGURE 8: The cumulative distribution function of capacity at Location 3.

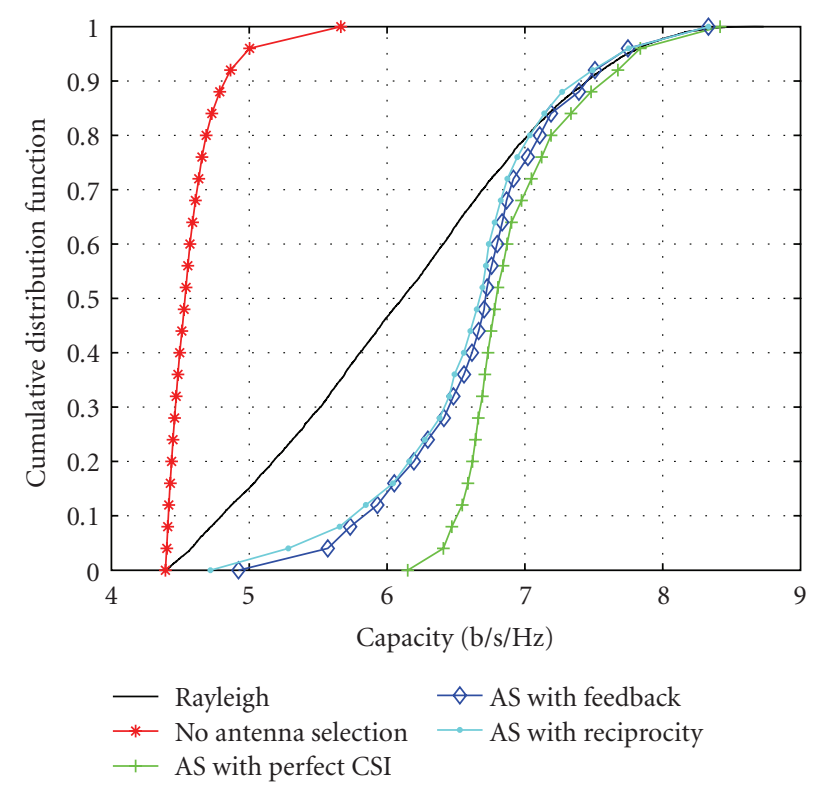

FIGURE 9: The cumulative distribution function of capacity at Location 4.

between reciprocity and feedback approaches is larger than Locations 1 and 2. In this response, the authors generate the other cases of fixed transmitting antennas to closely investigate this outcome. The presented dot line with $(x, y)$ is defined as a system with fixed antennas which always use $x$ th and $y$ th transmitting antennas to perform $2 \times 2 \mathrm{MIMO}$ operation. The results indicate that a reciprocity case still offers a higher capacity than all cases of fixed antennas. It is implied that the AS method still works very well based on a reciprocity channel, but the channels at this location do not offer enough correlation to produce a high capacity even when the best subset is selected. In addition, AS performance at Location 3 is lower than other locations. This is because the channels at this location act as high correlated channels due to LOS signals illustrated in Figure 1. 


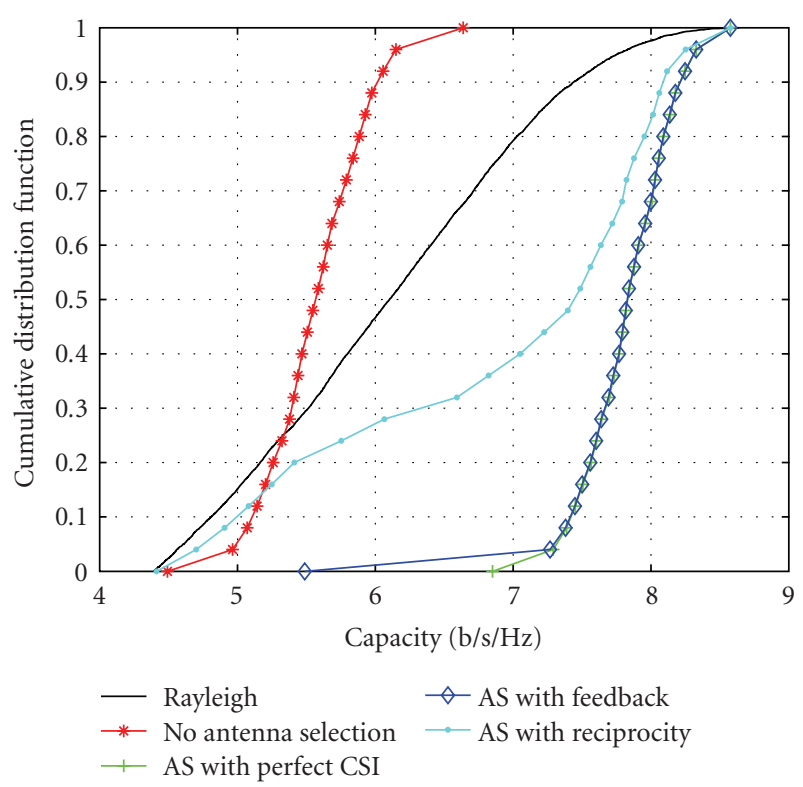

Figure 10: The cumulative distribution function of capacity at Location 5.

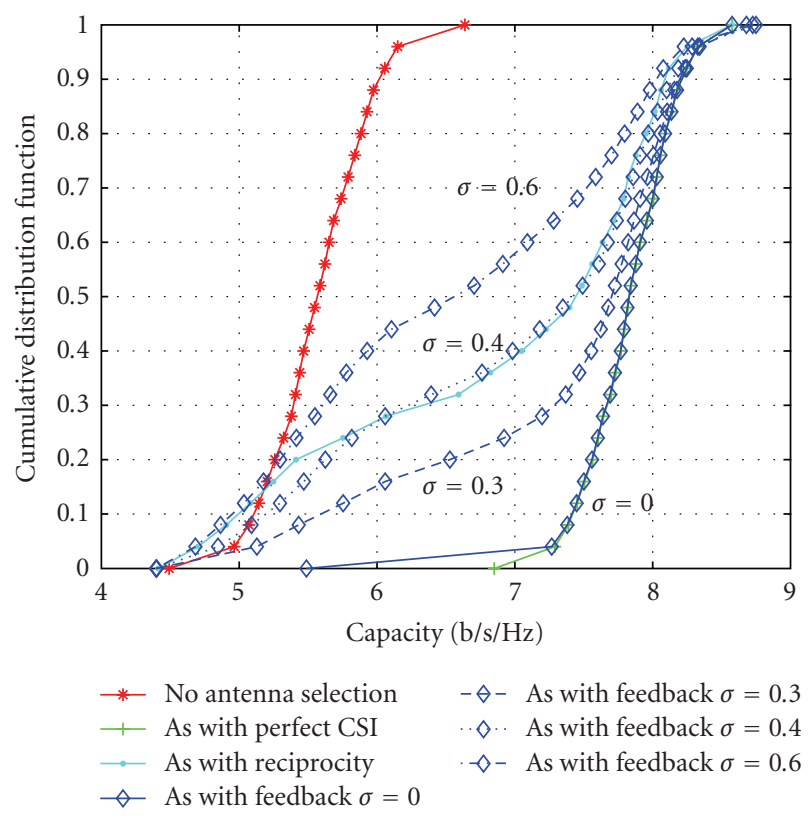

FIGURE 11: The cumulative distribution function of capacity with the presence of feedback error at Location 5.

Location 4. The aim of measuring at this location is to investigate the channel property when there are obstructions between transmitter and receiver, in comparison with Location 3. As shown in Figure 9, the cumulative distribution functions of capacity for AS methods with reciprocity and feedback cases are similar and close to a perfect CSI case after $50 \%$ probability. The capacity results of AS methods in this location are higher than the results at Location 3. For Location 4, the use of AS method with reciprocity can provide $2.2 \mathrm{bps} / \mathrm{Hz}$ higher than a case of fixed antennas method at $50 \%$ probability.
Location 5. As noticed in Figure 10, the results of AS method with reciprocity seem to fail on selecting the best subset of antennas. However, the authors did the other cases of fixed transmitting antennas and achieved the same conclusion that AS method with reciprocity still provides the highest capacity over all cases of fixed antennas.

In summary, a system using AS method with reciprocity always gives better performance better than fixed antennas while the feedback and perfect CSI cases slightly outperform this method. However, for AS method with feedback, the presented results are based on the exclusion of any errors in a feedback channel in which these errors are compulsorily occurred in practice due to channel delays and feedback noises. As a result, it is also necessary to examine the effect of feedback errors on capacity performances.

In order to investigate feedback errors included in feedback channel, this work assumes that the model of feedback error $\left(\varepsilon_{F}\right.$ from $\left.(3)\right)$ is given by

$$
\varepsilon_{F}=\sigma \mathbf{H}_{\text {i.i.d. }}
$$

where $\mathbf{H}_{\text {i.i.d. }}$ is i.i.d. (independent identically distributed) channel matrix with zero mean and unit variance $\sigma^{2}$ is the variance of feedback errors $\varepsilon_{F}$.

Figure 11 shows the effect of feedback errors on capacity performance of AS method at Location 5. It is obviously seen that errors degrade the capacity performance as a function of error variance. Also seen in this figure, the capacity performance of AS method with feedback is worse than a reciprocity case when the error variance in a feedback channel is more than 0.4. The results indicate the tradeoffs between using reciprocity and feedback approaches. If the variance of feedback errors is higher than 0.4 , the reciprocity approach might be more attractive than feedback with the benefit of low complexity.

\section{Conclusion}

This paper presents the performance of an adaptive $2 \times 4$ MIMO system when AS technique is employed at transmitter using channel reciprocity realized by the measured data. The experimental results reveal that antenna selection using channel reciprocity provides the capacity performance slightly worse than perfectly knowing CSI at transmitter. Furthermore, the system using AS method with reciprocity approach offers higher capacity than system with fixed antennas for all locations. Instead of feedback approach, the reciprocity does not require any information sent back to the other side. Therefore, the proposed system can properly be an attractive choice to replace the feedback system with the less complexity.

\section{Acknowledgments}

This work is financially supported by Thailand Research Fund (Grant no. MRG 5180344). The authors are also thankful to TRIDI as one of the authors received NTC scholarship funded by TRIDI. 


\section{References}

[1] R. D. Murch and K. Ben Letaief, "Antenna systems for broadband wireless access," IEEE Communications Magazine, vol. 40, no. 4, pp. 76-83, 2002.

[2] K. K. Wong, R. D. Murch, and K. B. Letaief, "Performance enhancement of multiuser MIMO wireless communication systems," IEEE Transactions on Communications, vol. 50, no. 12, pp. 1960-1970, 2002.

[3] G. J. Foschini and M. J. Gans, "On limits of wireless communications in a fading environment when using multiple antennas," Wireless Personal Communications, vol. 6, no. 3, pp. 311-335, 1998.

[4] I. E. Telatar, Capacity of Multiantenna Gaussian Channels, AT\&T Bell Laboratories, Technical Memorandum, 1995.

[5] N. Kong and L. B. Milstein, "Combined average SNR of a generalized diversity selection combining scheme," in Proceedings of the IEEE International Conference on Communications (ICC '98), vol. 3, pp. 1556-1560, June 1998.

[6] M. Z. Win and J. H. Winters, "Analysis of hybrid selection/maximal-ratio combining in rayleigh fading," IEEE Transactions on Communications, vol. 47, no. 12, pp. 17731776, 1999.

[7] M. Z. Win and J. H. Winters, "Analysis of hybrid selection/maximal-ratio combining of diversity branches with unequal SNR in Rayleigh fading," in Proceedings of the 49th IEEE Vehicular Technology Conference, pp. 215-220, May 1999.

[8] M. Z. Win, N. C. Beaulieu, L. A. Shepp, B. F. Logan, and J. H. Winters, "On the SNR penalty of MPSK with hybrid selection/maximal ratio combining over IID Rayleigh fading channels," IEEE Transactions on Communications, vol. 51, no. 6, pp. 1012-1023, 2003.

[9] A. F. Molisch, M. Z. Win, and J. H. Winters, "Capacity of MIMO systems with antenna selection," in Proceedings of the IEEE International Conference on Communications (ICC '01), pp. 570-574, June 2001.

[10] R. Nabar, D. Gore, and A. Paulrai, "Optimal selection and use of transmit antennas in wireless systems," in IEEE International Conference on Telecommunications, 2000.

[11] R. S. Blum and J. H. Winters, "On optimum MIMO with antenna selection," IEEE Communications Letters, vol. 6, no. 8, pp. 322-324, 2002.

[12] L. Dai, S. Sfar, and K. B. Letaief, "Optimal antenna selection based on capacity maximization for MIMO systems in correlated channels," IEEE Transactions on Communications, vol. 54, no. 3, pp. 563-573, 2006.

[13] R. W. Heath and A. Paulraj, "Antenna selection for spatial multiplexing systems based on minimum error rate," in Proceedings of the IEEE International Conference on Communications (ICC '00), pp. 2276-2280, June 2000.

[14] A. Ghrayeb and T. M. Duman, "Performance analysis of MIMO systems with antenna selection over quasi-static fading channels," IEEE Transactions on Vehicular Technology, vol. 52, no. 2, pp. 281-288, 2003.

[15] Y. Zhang, G. Zheng, C. Ji, K. K. Wong, D. J. Edwards, and T. Cui, "Near-optimal joint antenna selection for amplify-andforward relay networks," in Proceedings of the International Conference on Wireless Communications and Signal Processing, pp. 1-5, November 2009.

[16] J. C. Guey and L. D. Larsson, "Modeling and evaluation of MIMO systems exploiting channel reciprocity in TDD mode," in Proceedings of the 60th IEEE Vehicular Technology Conference, vol. 6, pp. 4265-4269, September 2004.
[17] A. Tölli and M. Codreanu, "Compensation of interference non-reciprocity in adaptive TDD MIMO-OFDM systems," in Proceedings of the 15th IEEE International Symposium on Personal, Indoor and Mobile Radio Communications, vol. 2, pp. 859-863, September 2004.

[18] D. Kim and M. Torlak, "Rapid prototyping of a cost effective and flexible 4X4 MIMO testbed," in Proceedings of the 5th IEEE Sensor Array and Multichannel Signal Processing Workshop, pp. 5-8, July 2008.

[19] K. Nishimori, R. Kudo, N. Honma, Y. Takatori, O. Atsushi, and K. Okada, "Experimental evaluation using $16 \times 16$ Multiuser MIMO testbed in an actual indoor scenario," in Proceedings of the IEEE International Symposium on Antennas and Propagation, pp. 1-4, July 2008.

[20] F. Azami, A. Ghorssi, H. Hemesi, A. Mohammadi, and A. Abdipour, "Design and implementation of a flexible $4 \times 4$ MIMO testbed," in Proceedings of the International Symposium on Telecommunications, pp. 268-272, August 2008.

[21] S. Chen, C. Jun, and T. Ohira, Handbook on Advancements in Smart Antenna Technologies for Wireless Networks, Information Science Reference, 2009.

[22] P. Uthansakul, K. Bialkowski, M. Bialkowski, and A. Postula, "Assessing an FPGA implemented mimo testbed with the use of channel emulator," in Proceedings of the 16th International Conference on Microwaves, Radar and Wireless Communications, pp. 410-413, May 2006.

[23] T. Onizawa, A. Ohta, and Y. Asai, "Experiments on FPGA-implemented eigenbeam MIMO-OFDM with transmit antenna selection," IEEE Transactions on Vehicular Technology, vol. 58, no. 3, pp. 1281-1291, 2009.

[24] P. Uthansakul, K. Attakitmongkol, N. Promsuvana, and M. Uthansakul, "MIMO antenna selections using CSI from reciprocal channel," WASET International Journal of Electrical and Information Engineering, vol. 5, pp. 349-358, 2010.

[25] J. Jose, A. Ashikhmin, P. Whiting, and S. Vishwanath, "Scheduling and pre-conditioning in multi-user MIMO TDD systems," in Proceedings of the IEEE International Conference on Communications (ICC '98), pp. 4100-4105, May 2008.

[26] A. J. Johansson, J. Karedal, F. Tufvesson, and A. F. Molisch, "MIMO channel measurements for personal area networks," in Proceedings of the 61st IEEE Vehicular Technology Conference, pp. 171-176, June 2005. 

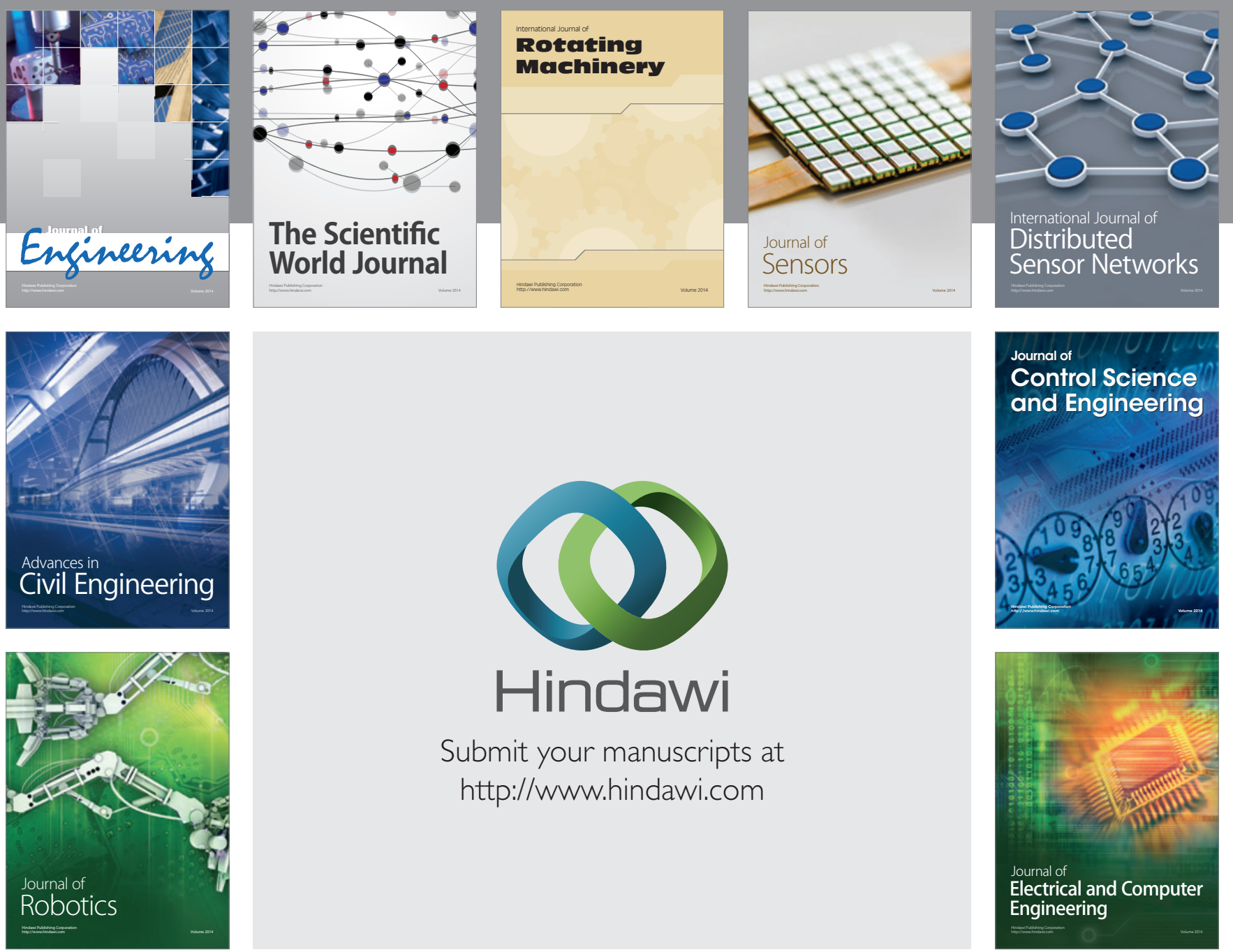

Submit your manuscripts at

http://www.hindawi.com
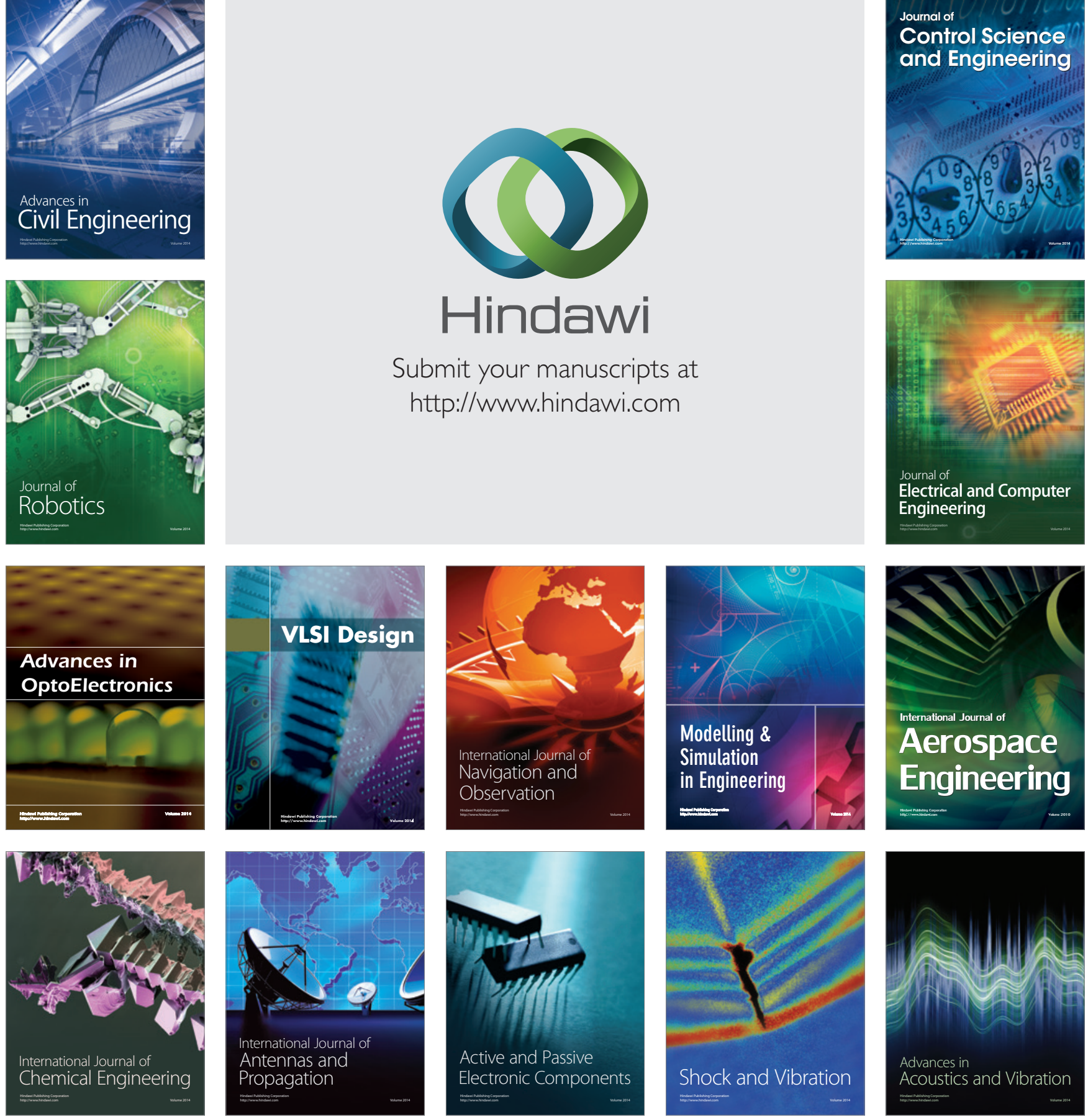